\title{
Generation of Heat Transfer Coefficient Data in Regenerator for Stirling Cycle Refrigeration System
}

\author{
Narendra. N .Wadaskar, S.K.Choudhary, R.D.Askhedkar
}

\begin{abstract}
This paper attempts to generate the data of heat transfer coefficient for regenerator in stirling cycle refrigeration system on the basis of available experimental data. The available data is based on assumption that the mode of heat transfer in regenerator is conduction. This data does not produce optimal design of regenerator. Heat transfer coefficient must be modified to account for heat transfer in a regenerator by all three modes i.e conduction, convection \& radiation. The data for heat transfer coefficient is generated on the basis of experimental results available in literature of performance of various stirling cycle refrigeration systems with different designs of regenerator. These models can be used to predict the performance of stirling cycle refrigerator system on the basis of dimensions of regenerator. The models are validated and optimized.
\end{abstract}

This paper also presents the effect of variations in regenerator dimensions i.e. regenerator length, regenerator diameter, wiremeshsize, wire mesh arrangement, and wire mesh materialonHeat Transfer coefficient of regenerator.

This data for heat transfer coefficient for regenerator of Stirling cycle refrigeration system can be used for optimizing design of regenerator of stirling cycle refrigeration system or predicting performance of stirling cycle system accurately. ,

Keywords: Regenerator, Performance, Regenerator length, Regenerator diameter; optimal value; Regenerator; Heat Transfer Coefficient.

\section{INTRODUCTION}

Presently vapour compression refrigeration systems are the most commonly used among all refrigeration systems. Cloro-Fluoro-Carbon (CFC) is used as a refrigerant in this system, this refrigerant $\mathrm{CFC}$ are most destructive to environment as they caused depletion of stratospheric ozone layer and contribute to the green house global warming. Our country is a party to Montreal protocol and Kyoto protocol. as per Montreal protocol The CFC group of refrigerant which cause ozone layer depletion should be banned by year 2010 and HCFC refrigerant do not cause ozone layer depletion but lead to global

Revised Manuscript Received on July 22, 2019.

Narendra .N .Wadaskar, Ph.D.Scholarin MechanicalEngineering, Nagpur University, Maharashtra INDIA.

Dr S.K.Choudhary, Prof.in Mechanical EnggDeptof K.D.K College of Engg,Nagpur, Maharashtra, INDIA.

Dr R.D.Askhedkar, Ex-Principal K.D.K College of Engg, Nagpur, Ex-Professor V.R.C.E Nagpur, INDIA. warming.kyotoprotocol is signed in 1997 and banned used of HCFC as a refrigerant to prevent global warming by year 2030.An alternate refrigeration system is working on non CFC, non HCFC and environment friendly refrigerant should be used.

\subsection{DRAWBACKS OF PRESENT VAPOUR COMPRESSION REFRIGERATION SYSTEM}

1. The heat transfer through refrigerants in VCRS is in the form of latent heat; so the refrigerants used in this system should have the property to change their phase at the desired pressure and temperature conditions. The refrigerant having the above properties is Cloro-Fluoro-Carbon (CFC). The CFC refrigerants have very high ozone depletion potential and also cause global warming.

2. High initial cost.

3. CFC refrigerant are hazardous for environment.

\subsection{NECESSITY OF NEW REFRIGERATION SYSTEM}

Chlorofluorocarbons (CFCs) have been used extensively in last five or six decades as refrigerants in the vapor compression cycle to produce refrigerating and air-conditioning effects. In recent years it has been found that CFCs are most destructive to the environment. It has been proved that CFCs are a major cause of depletion of the earth's stratospheric ozone layer and contribute to the greenhouse effect (global warming).

Presently large quantities of CFCs are being used as refrigerants in a number of refrigerating and air-conditioning systems. Though the refrigerant moves in a closed cycle, there are lots of leakages that escape to the atmosphere and cause destruction of the ozone layer. The most shocking fact about CFCs is that they have exceptionally long atmospheric life which, in certain cases, even extends to 100 years. This means that if CFC refrigerants are leaked today in the atmosphere, they will keep depleting ozone layer for the next 100 years.

When the CFC refrigerants are leaked from refrigeration or air-conditioning systems, they drift around the lower layers of the atmosphere. Slowly they start infiltrating into the upper layers

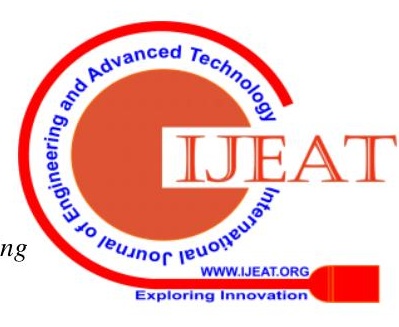


of the atmosphere and soon reach the ozone rich stratosphere, where they undergo major chemical changes.

In the ozone layer, sunlight enters in its pristine pure form; it is called ultraviolet radiation, which is highly intense and dangerous to plant and human life. The ozone layer filters this highly intense sunlight and allows less intense sunlight, which is not harmful to human and plant life, to the surface of the earth.

The unfiltered sunlight bombards the molecules of CFC refrigerant and they are pushed towards the stratospheric clouds over the poles. Due to this the CFC molecules get disintegrated. The chlorine atom removed from CFCs reacts with ozone molecule (O3) and converts it into oxygen molecule $(\mathrm{O} 2)$.

Now, the oxygen does not have the capacity to filter the highly intense ultraviolet radiations. So what is happening because of the CFC refrigerants is that the protective ozone layer is getting converted into incapable oxygen. Due to this the amounts of ultraviolet rays reaching the surface of the earth becomes very high and then causes excessive heating in the environment, called the greenhouse effect.

\section{SET UP OF $\alpha$ CONFIGURATION STIRLING CYCLE REFRIGERATION SYSTEM}

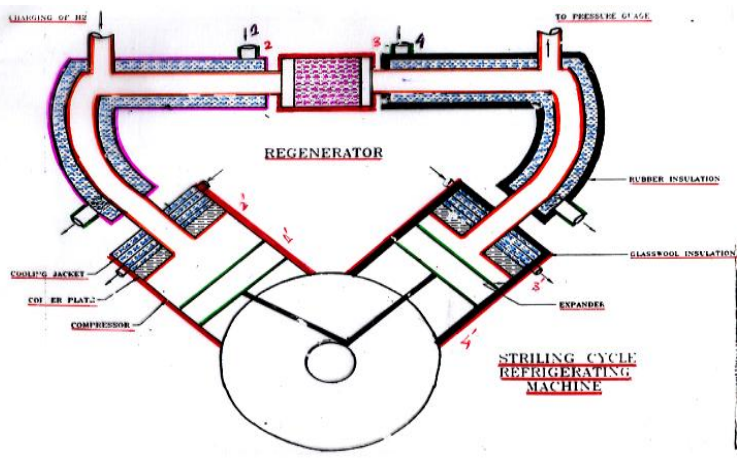

Fig.No.1

In starting the system is charged at predetermined pressure by compressor. The output of this compressor is connected to the air supply of the system. In cylinder 1 air is compressed and the compressed air is passes through the tube to regenerator consisting of a copper tube with copper mesh compacted inside the regenerator, due to the compression of the air the temp increases. The temperature at the entry of the regenerator is designated as $T_{2}$ and the temp of outlet is designated as $T_{3}$. As the hot air passes through the regenerator, it transfers the heat in the copper mesh and cools. The temp at the exit point is Designated as $\mathrm{T}_{4}$ which is less than the temp at the inlet i.e. $\mathrm{T}_{2}$, it passes through the tube goes to the expander. The tube connecting the regenerator and the expander is covered by water jacket through its water circulated where it cools the air further.
This heat exchanger reduce the temp of air .The cooled refrigerant is displaced form expander through cooling water Jacket and double pipe heat exchanger where refrigerant absorbs heat from the water flowing through the water Jacket and double pipe heat exchanger to regenerator where it absorbs heat from wire mesh and heated refrigerant passes through the double pipe heat exchanger and water Jacket to compressor, which completes the cycle. The cycle goes on repeating and the temp $T_{3}$ and $T_{2}$ get stabilize after some time, after that stabilization the temperature of the refrigerating machine remains constant.

\subsection{STEPS FOR CALCULATION HTC AND COP}

\section{Step 1: Find out the Mass Flow Rate.}

Bore Diameter (D) of Cylinder $=70 \mathrm{~mm}$

Total Length of Cylinder $=85 \mathrm{~mm}$

$\mathrm{M}^{\prime}=\mathrm{Q} \times \rho_{\mathrm{air}}$

Step 2 : Find out the Reynolds Number.

$\mathrm{R}_{\mathrm{e}}=\frac{4 \mathrm{~m}^{r}}{\mathrm{nd} \mu}$

If $\mathrm{R}_{\mathrm{e}}<\mathbf{2 3 0 0 f l o w}$ is Laminar

If $R_{e}>2300 f l o w$ is Turbulent

Step 3: Find out the Nusselt Number.

$\mathrm{N}_{\mathrm{u}}=0.023\left(\mathrm{R}_{\mathrm{e}}\right)^{0.3} \times\left(\mathrm{P}_{\mathrm{r}}\right)^{\mathrm{n}}$

$\mathrm{n}=0.4$ when fluid is being heated

$\mathrm{n}=0.3$ when fluid is being cold

Step 4 : Calculate the Heat Transfer Coefficient of air passing through Regenerator of $35 \mathrm{~mm}$ Diameter filled with copper mesh wire.

$\mathrm{N}_{\mathrm{u}}=\frac{\mathrm{hD}}{\mathrm{k}}$

$\mathrm{h}=\mathrm{k} \mathrm{N}_{\mathrm{u}} / \mathrm{D}$

Step 5 : Calculate the Heat Transfer.

$\mathrm{Q}_{\mathrm{L}}=\mathrm{m}_{\mathrm{w}} \mathrm{Cp}_{\mathrm{w}} \Delta \mathrm{T}_{\mathrm{w}}$

Where $\mathrm{Q}_{\mathrm{L}}$ is heat lifted in $\mathrm{J} / \mathrm{sec}$

$\mathrm{m}_{\mathrm{w}}$ is the mass of water in the insulated reservoir.

$\Delta \mathrm{T}_{\mathrm{w}}$ is the drop in temperature of reservoir water during

experimentation

$\mathrm{Q}_{\mathrm{L}}=\mathrm{m}_{\mathrm{w}} \mathrm{C} \mathrm{p}_{\mathrm{w}}$ (T3-T4)

$\mathrm{Q}$

$=\mathrm{h} \mathrm{A} \Delta \mathrm{T}$

Step 6 : Calculate Work Done

$\mathrm{W}=\mathrm{P}_{1} \mathrm{~V}_{1} \ln P \max / P \min$

Step 6: Calculate COP.

$$
\mathrm{COP}=\frac{Q}{W}=4.476
$$

\section{PLANNING OF EXPERIMENTATION}

Classical plan of experimentation was used for conducting experimentation on stirling cycle refrigeration system. the design parameters of regenerator are varied over largest possible range. To reduce the variables in experimentation dimension analysis was carried out. The total experimentation was carried 
out at 25 test points. The table 1 shows the experimental reading at 25 setting of the machines.
The experimental results obtained are shown in Table No.1

Table No.1 Result table for change in $\pi_{1}, \pi_{2}, \pi_{3}, \pi_{4}$ \& $\pi_{5}$

\begin{tabular}{|c|c|c|c|c|c|c|c|c|c|c|}
\hline Sr.No & $\pi_{1 / /} L_{\mathbf{R}(\mathbf{m m})}$ & $\pi_{2 /} \mathrm{D}_{\mathrm{R}(\mathrm{mm})}$ & $\pi_{3 /} \mathrm{D}_{\mathrm{M}(\mathrm{mm})}$ & $\pi_{4 /} \mathrm{V}_{\mathbf{R}(\mathrm{mm} 3)}$ & $\pi_{5 / \mathbf{P}(\mathrm{psi})}$ & $\mathbf{W}$ & $\mathbf{T} 1^{\circ} \mathrm{C}$ & $\mathrm{T} 4^{\circ} \mathrm{C}$ & $\mathrm{COP}_{\mathrm{TE}}$ & $\mathrm{COP}_{\mathrm{A}}$ \\
\hline 1 & $2.13 / 105$ & $0.91 / 45$ & $0.01826 / 0.3$ & $0.216 / 139060$ & $0.156 / 50$ & 270 & 49 & 10 & 7.256 & 0.9284 \\
\hline 2 & $2.434 / 120$ & $0.91 / 45$ & $0.01826 / 0.3$ & $0.216 / 139060$ & $0.156 / 50$ & 285 & 44 & 10 & 8.3235 & 1.2144 \\
\hline 3 & $2.738 / 135$ & $0.91 / 45$ & $0.01826 / 0.3$ & $0.216 / 139060$ & $0.156 / 50$ & 290 & 38 & 10 & 9.4330 & 1.6091 \\
\hline 4 & $3.043 / 150$ & $0.91 / 45$ & $0.01826 / 0.3$ & $0.216 / 139060$ & $0.156 / 50$ & 335 & 38 & 10 & 10.481 & 1.816 \\
\hline 5 & $3.347 / 165$ & $0.91 / 45$ & $0.01826 / 0.3$ & $0.216 / 139060$ & $0.156 / 50$ & 355 & 35 & 10 & 11.32 & 2.0433 \\
\hline 6 & $2.738 / 135$ & $0.71 / 35$ & $0.01826 / 0.3$ & $0.216 / 139060$ & $0.156 / 50$ & 310 & 44 & 10 & 7.075 & 0.8045 \\
\hline 7 & $2.738 / 135$ & $0.81 / 40$ & $0.01826 / 0.3$ & $0.216 / 139060$ & $0.156 / 50$ & 320 & 44 & 10 & 8.323 & 1.2378 \\
\hline 8 & $2.738 / 135$ & $0.91 / 45$ & $0.01826 / 0.3$ & $0.216 / 139060$ & $0.156 / 50$ & 315 & 40 & 10 & 9.433 & 1.6091 \\
\hline 9 & $2.738 / 135$ & $1.01 / 50$ & $0.01826 / 0.3$ & $0.216 / 139060$ & $0.156 / 50$ & 310 & 37 & 10 & 10.482 & 1.84 \\
\hline 10 & $2.738 / 135$ & $1.11 / 55$ & $0.01826 / 0.3$ & $0.216 / 139060$ & $0.156 / 50$ & 300 & 34 & 10 & 11.79 & 2.228 \\
\hline 11 & $2.738 / 135$ & $0.91 / 45$ & $0.0183 / 0.1$ & $0.216 / 139060$ & $0.156 / 50$ & 305 & 40 & 10 & 9.433 & 1.6091 \\
\hline 12 & $2.738 / 135$ & $0.91 / 45$ & $0.00406 / 0.2$ & $0.216 / 139060$ & $0.156 / 50$ & 310 & 41 & 10 & 9.129 & 1.34 \\
\hline 13 & $2.738 / 135$ & $0.91 / 45$ & $0.00609 / 0.3$ & $0.216 / 139060$ & $0.156 / 50$ & 305 & 42 & 10 & 8.84 & 1.11 \\
\hline 14 & $2.738 / 135$ & $0.91 / 45$ & $0.00811 / 0.4$ & $0.216 / 139060$ & $0.156 / 50$ & 305 & 43 & 10 & 8.57 & 0.847 \\
\hline 15 & $2.738 / 135$ & $0.91 / 45$ & $0.01014 / 0.5$ & $0.216 / 139060$ & $0.156 / 50$ & 300 & 44 & 10 & 8.32 & 0.585 \\
\hline 16 & $2.738 / 135$ & $0.91 / 45$ & $0.01826 / 0.3$ & $0.216 / 139060$ & $0.156 / 50$ & 305 & 40 & 10 & 9.433 & 1.609 \\
\hline 17 & $2.738 / 135$ & $0.91 / 45$ & $0.01826 / 0.3$ & $0.186 / 119746$ & $0.156 / 50$ & 310 & 41 & 10 & 9.129 & 1.399 \\
\hline 18 & $2.738 / 135$ & $0.91 / 45$ & $0.01826 / 0.3$ & $0.17 / 109316$ & $0.156 / 50$ & 305 & 44 & 10 & 8.843 & 1.156 \\
\hline 19 & $2.738 / 135$ & $0.91 / 45$ & $0.01826 / 0.3$ & $0.147 / 11619$ & $0.156 / 50$ & 305 & 44 & 10 & 8.5750 & 0.91 \\
\hline 20 & $2.738 / 135$ & $0.91 / 45$ & $0.01826 / 0.3$ & $0.123 / 79315$ & $0.156 / 50$ & 300 & 44 & 10 & 8.32 & 0.631 \\
\hline 21 & $2.738 / 135$ & $0.91 / 45$ & $0.01826 / 0.3$ & $0.216 / 139060$ & $0.195 / 40$ & 310 & 38 & 10 & 10.1 & 1.93 \\
\hline 22 & $2.738 / 135$ & $0.91 / 45$ & $0.01826 / 0.3$ & $0.216 / 139060$ & $0.1733 / 45$ & 305 & 39 & 10 & 9.758 & 1.787 \\
\hline 23 & $2.738 / 135$ & $0.91 / 45$ & $0.01826 / 0.3$ & $0.216 / 139060$ & $0.156 / 50$ & 305 & 40 & 10 & 9.433 & 1.6091 \\
\hline 24 & $2.738 / 135$ & $0.91 / 45$ & $0.01826 / 0.3$ & $0.216 / 139060$ & $0.142 / 55$ & 310 & 43 & 10 & 5.575 & 1.462 \\
\hline 25 & $2.738 / 135$ & $0.91 / 45$ & $0.01826 / 0.3$ & $0.216 / 139060$ & $0.13 / 60$ & 305 & 45 & 10 & 8.084 & 1.379 \\
\hline
\end{tabular}

\subsection{THEORETICAL EVALUATION OF} PERFORMANCE OF ALPHA CONFIGURATION STIRLING CYCLE REFRIGERATION SYSTEM

The performance of alpha configuration stirling cycle refrigeration systems with 25 different regenerators are evaluated by using fundamental of Heat Transfer. The theoretical coefficient of performance $\left(\mathrm{COP}_{\mathrm{T}}\right)$ for refrigeration systems are shown in table

Sample calculations for evaluating performance of stirling cycle refrigeration system with first regenerator (Sr.no 1 , Table no 2 are as given below)

Evaluation of mass flow $\operatorname{rate}(M)$ :

$\mathrm{Q}=\frac{\pi}{4}(\mathrm{D})^{2} \mathrm{~L} \frac{N}{60}$

Substituting

$\mathrm{D}=$ Bore Diameter of compressor cylinder $=70 \mathrm{~mm}$

$\mathrm{L}=$ Stroke of compressor $=85 \mathrm{~mm}$

$\mathrm{N}=$ number of strokes per minute $=750$.

$\mathrm{Q}=0.004 \mathrm{~m}^{3} / \mathrm{sec}$
Mass Flow Rate of Refrigerant (M)

$\mathrm{M}=\mathrm{Q} \times \rho_{\text {air }}$

Where $\rho_{\text {air }}=$ Density of air

Substituting $Q=0.004 \mathrm{~m}^{3} / \mathrm{sec}$ and $\rho_{\text {air }}=1.225 \mathrm{~kg} / \mathrm{m}^{3}$ $\mathrm{Q}=0.005 \mathrm{~kg} / \mathrm{sec}$

Evaluation of Heat Transfer Coefficient of air passing through Regenerator of $\mathbf{3 5} \mathbf{~ m m}$ Diameter filled with copper mesh wire.

$\mathrm{N}_{\mathrm{u}}=\frac{\mathrm{hD}}{\mathrm{k}}$

$\mathrm{h}=\mathrm{k} \mathrm{N}_{\mathrm{u}} / \mathrm{D}$

Evaluation of Heat Transfer(Q).

$\mathrm{Q}=\mathrm{h} \mathrm{A} \Delta \mathrm{T}$

Evaluation of Work Done(W)

$\mathrm{W}=\mathrm{P}_{1} \mathrm{~V}_{1} \ln P \max / P \min$

Evaluation of $\operatorname{COP}\left(\mathrm{COP}_{\mathrm{TH}}\right)$.

$\mathrm{COP}_{\mathrm{TH}}=\frac{Q}{W}=4.4765$

\subsection{EXPERIMENTAL AND \\ CALCULATED \\ THEORETICAL RESULTS}


The table 2 shows the calculated theoretical reading at $25 \quad$ setting of the machines.

Table No.2 Result table for change in $\pi_{1}, \pi_{2}, \pi_{3}, \pi_{4} \& \pi_{5}$

\begin{tabular}{|c|c|c|c|c|c|c|c|c|c|c|}
\hline Sr.No. & $\square_{\text {1/LR(mm) }}$ & $\begin{array}{l}\square_{\text {2/DR(m }} \\
\text { m) }\end{array}$ & $\square_{\text {3/DM }}$ & $\square_{\text {4/VR }}$ & $\square_{5 / \mathbf{P}}$ & $\mathbf{T}_{1}$ & $\mathbf{T}_{4}$ & $\mathrm{COP}_{\mathrm{TE}}$ & $\mathrm{COP}_{\mathrm{TH}}$ & $\begin{array}{c}\% \\
\text { Difference }\end{array}$ \\
\hline 1 & $2.13 / 105$ & $0.91 / 45$ & $0.01826 / 0.3$ & $0.216 / 139060$ & $0.156 / 50$ & 49 & 10 & 7.256 & 4.47665 & 38.3041 \\
\hline 2 & $2.434 / 120$ & $0.91 / 45$ & $0.01826 / 0.3$ & $0.216 / 139060$ & $0.156 / 50$ & 44 & 10 & 8.3235 & 4.38529 & 47.3144 \\
\hline 3 & $2.738 / 135$ & $0.91 / 45$ & $0.01826 / 0.3$ & $0.216 / 139060$ & $0.156 / 50$ & 38 & 10 & 9.433 & 3.94676 & 58.1600 \\
\hline 4 & $3.043 / 150$ & $0.91 / 45$ & $0.01826 / 0.3$ & $0.216 / 139060$ & $0.156 / 50$ & 38 & 10 & 10.481 & 4.38529 & 58.1616 \\
\hline 5 & $3.347 / 165$ & $0.91 / 45$ & $0.01826 / 0.3$ & $0.216 / 139060$ & $0.156 / 50$ & 35 & 10 & 11.32 & 4.22084 & 62.7134 \\
\hline 6 & $2.738 / 135$ & $0.71 / 35$ & $0.01826 / 0.3$ & $0.216 / 139060$ & $0.156 / 50$ & 50 & 10 & 7.075 & 5.92014 & 16.3230 \\
\hline 7 & $2.738 / 135$ & $0.81 / 40$ & $0.01826 / 0.3$ & $0.216 / 139060$ & $0.156 / 50$ & 44 & 10 & 8.323 & 4.93345 & 40.7250 \\
\hline 8 & $2.738 / 135$ & $0.91 / 45$ & $0.01826 / 0.3$ & $0.216 / 139060$ & $0.156 / 50$ & 40 & 10 & 9.433 & 4.27566 & 54.6733 \\
\hline 9 & $2.738 / 135$ & $1.01 / 50$ & $0.01826 / 0.3$ & $0.216 / 139060$ & $0.156 / 50$ & 37 & 10 & 10.482 & 3.78231 & 63.9161 \\
\hline 10 & $2.738 / 135$ & $1.11 / 55$ & $0.01826 / 0.3$ & $0.216 / 139060$ & $0.156 / 50$ & 34 & 10 & 11.79 & 3.28896 & 72.1037 \\
\hline 11 & $2.738 / 135$ & $0.91 / 45$ & $0.0183 / 0.1$ & $0.216 / 139060$ & $0.156 / 50$ & 40 & 10 & 9.433 & 4.27566 & 54.67339 \\
\hline 12 & $2.738 / 135$ & $0.91 / 45$ & $0.00406 / 0.2$ & $0.216 / 139060$ & $0.156 / 50$ & 41 & 10 & 9.129 & 4.44011 & 51.3626 \\
\hline 13 & $2.738 / 135$ & $0.91 / 45$ & $0.00609 / 0.3$ & $0.216 / 139060$ & $0.156 / 50$ & 42 & 10 & 8.84 & 4.60456 & 47.91226 \\
\hline 14 & $2.738 / 135$ & $0.91 / 45$ & $0.00811 / 0.4$ & $0.216 / 139060$ & $0.156 / 50$ & 43 & 10 & 8.57 & 4.76901 & 44.35223 \\
\hline 15 & $2.738 / 135$ & $0.91 / 45$ & $0.01014 / 0.5$ & $0.216 / 139060$ & $0.156 / 50$ & 44 & 10 & 8.32 & 4.93345 & 40.70369 \\
\hline 16 & $2.738 / 135$ & $0.91 / 45$ & $0.01826 / 0.3$ & $0.216 / 139060$ & $0.156 / 50$ & 40 & 10 & 9.433 & 4.27566 & 54.67339 \\
\hline 17 & $2.738 / 135$ & $0.91 / 45$ & $0.01826 / 0.3$ & $0.186 / 119746$ & $0.156 / 50$ & 41 & 10 & 9.129 & 4.44011 & 51.3626 \\
\hline 18 & $2.738 / 135$ & $0.91 / 45$ & $0.01826 / 0.3$ & $0.17 / 109316$ & $0.156 / 50$ & 42 & 10 & 8.843 & 4.60456 & 47.91226 \\
\hline 19 & $2.738 / 135$ & $0.91 / 45$ & $0.01826 / 0.3$ & $0.147 / 11619$ & $0.156 / 50$ & 44 & 10 & 8.575 & 4.76901 & 44.35223 \\
\hline 20 & $2.738 / 135$ & $0.91 / 45$ & $0.01826 / 0.3$ & $0.123 / 79315$ & $0.156 / 50$ & 44 & 10 & 8.32 & 4.93345 & 40.70369 \\
\hline 21 & $2.738 / 135$ & $0.91 / 45$ & $0.01826 / 0.3$ & $0.216 / 139060$ & $0.195 / 40$ & 38 & 10 & 10.1 & 3.94676 & 60.92315 \\
\hline 22 & $2.738 / 135$ & $0.91 / 45$ & $0.01826 / 0.3$ & $0.216 / 139060$ & $0.1733 / 45$ & 39 & 10 & 9.758 & 4.11121 & 57.8683 \\
\hline 23 & $2.738 / 135$ & $0.91 / 45$ & $0.01826 / 0.3$ & $0.216 / 139060$ & $0.156 / 50$ & 40 & 10 & 9.433 & 4.27566 & 54.67339 \\
\hline 24 & $2.738 / 135$ & $0.91 / 45$ & $0.01826 / 0.3$ & $0.216 / 139060$ & $0.142 / 55$ & 43 & 10 & 8.575 & 4.76901 & 44.38478 \\
\hline 25 & $2.738 / 135$ & $0.91 / 45$ & $0.01826 / 0.3$ & $0.216 / 139060$ & $0.13 / 60$ & 45 & 10 & 8.084 & 5.0979 & 36.93838 \\
\hline
\end{tabular}

\subsection{THEORETICAL EVALUATION OF HEAT TRANSFER COEFFICIENT ON THE BASIS OF EXPERIMENTAL \\ RESULTS FOR TEMP BASED. \\ 2) $\mathrm{Q}=\mathrm{hA}(\mathrm{Tmax}-\mathrm{Tmin}), \mathrm{Q}=\mathrm{h}\left(\pi^{*} \mathrm{D} * \mathrm{~L}\right)(\Delta \mathrm{T})$ \\ Where $\mathrm{D}=$ Diameter of Regenerator $=45 \mathrm{~mm}, \quad \mathrm{~L}=$ Length of Regenerator $=105 \mathrm{~mm}$}

Sample calculations for evaluating performance of stirling cycle refrigeration system with first regenerator (Sr.no 1,Table no 3.are as given below)

Evaluation of Heat Transfer(h).

1) $\mathrm{COP}_{\mathrm{TE}}=\mathrm{Q} / \mathrm{W}, \mathrm{Q}=\mathrm{COP}_{\mathrm{TE}} * \mathrm{~W}$
The table 3 shows the calculated heat transfer coefficient on the basis of experimental results for temp based reading at 25 setting of the machines.

Table No.3 Result table for change in $\pi_{1}, \pi_{2}, \pi_{3}, \pi_{4} \& \pi_{5}$

\begin{tabular}{|c|c|c|c|c|c|c|c|c|c|}
\hline Sr.No & $\pi_{1 / \mathrm{LR}(\mathrm{mm})}$ & $\pi_{2 / \mathrm{DR}(\mathrm{mm})}$ & $\pi_{3 / \mathrm{DM}(\mathrm{mm})}$ & $\pi_{4 / \mathrm{Vr}(\mathbf{m m} 3)}$ & $\pi_{5 / \mathbf{P}(\mathbf{p s i})}$ & $\operatorname{Tmax}^{0} K$ & $\operatorname{Tmin}^{0} \mathrm{~K}$ & $\mathrm{COP}_{\mathrm{TE}}$ & $\mathrm{HTC}_{\mathrm{TE}}$ \\
\hline 1 & $2.13 / 105$ & $0.91 / 45$ & $0.01826 / 0.3$ & $0.216 / 139060$ & $0.156 / 50$ & 322 & 273 & 7.256 & 3464.87 \\
\hline 2 & $2.434 / 120$ & $0.91 / 45$ & $0.01826 / 0.3$ & $0.216 / 139060$ & $0.156 / 50$ & 317 & 273 & 8.3235 & 3577.67 \\
\hline 3 & $2.738 / 135$ & $0.91 / 45$ & $0.01826 / 0.3$ & $0.216 / 139060$ & $0.156 / 50$ & 311 & 273 & 9.4330 & 3773.88 \\
\hline 4 & $3.043 / 150$ & $0.91 / 45$ & $0.01826 / 0.3$ & $0.216 / 139060$ & $0.156 / 50$ & 311 & 273 & 10.4815 & 3923.68 \\
\hline 5 & $3.347 / 165$ & $0.91 / 45$ & $0.01826 / 0.3$ & $0.216 / 139060$ & $0.156 / 50$ & 308 & 273 & 11.32 & 4029.31 \\
\hline 6 & $2.738 / 135$ & $0.71 / 35$ & $0.01826 / 0.3$ & $0.216 / 139060$ & $0.156 / 50$ & 317 & 273 & 7.075 & 3359.73 \\
\hline 7 & $2.738 / 135$ & $0.81 / 40$ & $0.01826 / 0.3$ & $0.216 / 139060$ & $0.156 / 50$ & 317 & 273 & 8.323 & 3569.88 \\
\hline 8 & $2.738 / 135$ & $0.91 / 45$ & $0.01826 / 0.3$ & $0.216 / 139060$ & $0.156 / 50$ & 313 & 273 & 9.433 & 3894.36 \\
\hline
\end{tabular}


International Journal of Engineering and Advanced Technology (IJEAT)

ISSN: 2249 - 8958, Volume-8, Issue-6S August 2019

\begin{tabular}{|c|c|c|c|c|c|c|c|c|c|}
\hline 9 & $2.738 / 135$ & $1.01 / 50$ & $0.01826 / 0.3$ & $0.216 / 139060$ & $0.156 / 50$ & 310 & 273 & 10.482 & 4143.53 \\
\hline 10 & $2.738 / 135$ & $1.11 / 55$ & $0.01826 / 0.3$ & $0.216 / 139060$ & $0.156 / 50$ & 307 & 273 & 11.79 & 4462 \\
\hline 11 & $2.738 / 135$ & $0.91 / 45$ & $0.0183 / 0.1$ & $0.216 / 139060$ & $0.156 / 50$ & 313 & 273 & 9.433 & 3770.63 \\
\hline 12 & $2.738 / 135$ & $0.91 / 45$ & $0.00406 / 0.2$ & $0.216 / 139060$ & $0.156 / 50$ & 314 & 273 & 9.129 & 3618.47 \\
\hline 13 & $2.738 / 135$ & $0.91 / 45$ & $0.00609 / 0.3$ & $0.216 / 139060$ & $0.156 / 50$ & 315 & 273 & 8.84 & 3365.32 \\
\hline 14 & $2.738 / 135$ & $0.91 / 45$ & $0.00811 / 0.4$ & $0.216 / 139060$ & $0.156 / 50$ & 316 & 273 & 8.57 & 3186.66 \\
\hline 15 & $2.738 / 135$ & $0.91 / 45$ & $0.01014 / 0.5$ & $0.216 / 139060$ & $0.156 / 50$ & 317 & 273 & 8.32 & 2973.83 \\
\hline 16 & $2.738 / 135$ & $0.91 / 45$ & $0.01826 / 0.3$ & $0.216 / 139060$ & $0.156 / 50$ & 313 & 273 & 9.433 & 3832.44 \\
\hline 17 & $2.738 / 135$ & $0.91 / 45$ & $0.01826 / 0.3$ & $0.186 / 119746$ & $0.156 / 50$ & 314 & 273 & 9.129 & 3560.11 \\
\hline 18 & $2.738 / 135$ & $0.91 / 45$ & $0.01826 / 0.3$ & $0.17 / 109316$ & $0.156 / 50$ & 317 & 273 & 8.84 & 3213.44 \\
\hline 19 & $2.738 / 135$ & $0.91 / 45$ & $0.01826 / 0.3$ & $0.147 / 11619$ & $0.156 / 50$ & 317 & 273 & 8.57 & 3167.14 \\
\hline 20 & $2.738 / 135$ & $0.91 / 45$ & $0.01826 / 0.3$ & $0.123 / 79315$ & $0.156 / 50$ & 317 & 273 & 8.32 & 3023.39 \\
\hline 21 & $2.738 / 135$ & $0.91 / 45$ & $0.01826 / 0.3$ & $0.216 / 139060$ & $0.195 / 40$ & 311 & 273 & 10.1 & 4319.4 \\
\hline 22 & $2.738 / 135$ & $0.91 / 45$ & $0.01826 / 0.3$ & $0.216 / 139060$ & $0.173 / 45$ & 312 & 273 & 9.758 & 3934.97 \\
\hline 23 & $2.738 / 135$ & $0.91 / 45$ & $0.01826 / 0.3$ & $0.216 / 139060$ & $0.156 / 50$ & 313 & 273 & 9.433 & 3832.44 \\
\hline 24 & $2.738 / 135$ & $0.91 / 45$ & $0.01826 / 0.3$ & $0.216 / 139060$ & $0.142 / 55$ & 316 & 273 & 5.575 & 3763.5 \\
\hline 25 & $2.738 / 135$ & $0.91 / 45$ & $0.01826 / 0.3$ & $0.216 / 139060$ & $0.13 / 60$ & 318 & 273 & 8.084 & 3390.32 \\
\hline
\end{tabular}

3.5 EVALUATION OF HEAT TRANSFER COEFFICIENT ON THE BASIS OF EXPERIMENTAL RESULTS FOR ACTUAL BASED

Sample calculations for evaluating performance of stirling cycle refrigeration system with first regenerator (Sr.no 1, Table no 4 are as given below)

Evaluation of Heat Transfer coefficient (h)

1) $Q=m_{w} C p_{w} \Delta T w$

\section{2) 2) $Q=h A T_{S}$}

The table 4 shows the calculated heat transfer coefficient on the basis of experimental results for actual based reading at 25 setting of the machines.

Table No.4 Result table for change in $\pi_{1}, \pi_{2}, \pi_{3}, \pi_{4} \& \pi_{5}$

\begin{tabular}{|c|c|c|c|c|c|c|c|c|}
\hline Sr.No & $\pi_{\mathbf{1} / \mathbf{L R}(\mathbf{m m})}$ & $\pi_{2 / \mathbf{D R}(\mathbf{m m})}$ & $\pi_{3 / \mathbf{I M}(\mathbf{m m})}$ & $\pi_{\mathbf{4} / \mathbf{V} \mathbf{r}(\mathbf{m m} \mathbf{3})}$ & $\pi_{\mathbf{5} / \mathbf{P}(\mathbf{p s i})}$ & $\Delta \mathbf{T W}^{\circ} \mathbf{C}$ & $\mathbf{C O P}_{\mathbf{A}}$ & $\mathbf{H T C}_{\mathbf{A}}$ \\
\hline 1 & $2.13 / 105$ & $0.91 / 45$ & $0.01826 / 0.3$ & $0.216 / 139060$ & $0.156 / 50$ & 3 & 0.9284 & 3.895936 \\
\hline 2 & $2.434 / 120$ & $0.91 / 45$ & $0.01826 / 0.3$ & $0.216 / 139060$ & $0.156 / 50$ & 4 & 1.2144 & 4.328818 \\
\hline 3 & $2.738 / 135$ & $0.91 / 45$ & $0.01826 / 0.3$ & $0.216 / 139060$ & $0.156 / 50$ & 6 & 1.6091 & 4.86992 \\
\hline 4 & $3.043 / 150$ & $0.91 / 45$ & $0.01826 / 0.3$ & $0.216 / 139060$ & $0.156 / 50$ & 7 & 1.816 & 5.681573 \\
\hline 5 & $3.347 / 165$ & $0.91 / 45$ & $0.01826 / 0.3$ & $0.216 / 139060$ & $0.156 / 50$ & 8 & 2.0433 & 5.771757 \\
\hline 6 & $2.738 / 135$ & $0.71 / 35$ & $0.01826 / 0.3$ & $0.216 / 139060$ & $0.156 / 50$ & 2 & 0.8045 & 3.2466 \\
\hline 7 & $2.738 / 135$ & $0.81 / 40$ & $0.01826 / 0.3$ & $0.216 / 139060$ & $0.156 / 50$ & 4 & 1.2378 & 4.328818 \\
\hline 8 & $2.738 / 135$ & $0.91 / 45$ & $0.01826 / 0.3$ & $0.216 / 139060$ & $0.156 / 50$ & 6 & 1.6091 & 4.86992 \\
\hline 9 & $2.738 / 135$ & $1.01 / 50$ & $0.01826 / 0.3$ & $0.216 / 139060$ & $0.156 / 50$ & 7 & 1.84 & 5.050287 \\
\hline 10 & $2.738 / 135$ & $1.11 / 55$ & $0.01826 / 0.3$ & $0.216 / 139060$ & $0.156 / 50$ & 9 & 2.228 & 5.31264 \\
\hline 11 & $2.738 / 135$ & $0.91 / 45$ & $0.0183 / 0.1$ & $0.216 / 139060$ & $0.156 / 50$ & 6 & 1.6091 & 4.86992 \\
\hline 12 & $2.738 / 135$ & $0.91 / 45$ & $0.00406 / 0.2$ & $0.216 / 139060$ & $0.156 / 50$ & 5 & 1.34 & 4.638019 \\
\hline 13 & $2.738 / 135$ & $0.91 / 45$ & $0.00609 / 0.3$ & $0.216 / 139060$ & $0.156 / 50$ & 4 & 1.11 & 4.328818 \\
\hline 14 & $2.738 / 135$ & $0.91 / 45$ & $0.00811 / 0.4$ & $0.216 / 139060$ & $0.156 / 50$ & 3 & 0.847 & 3.895936 \\
\hline 15 & $2.738 / 135$ & $0.91 / 45$ & $0.01014 / 0.5$ & $0.216 / 139060$ & $0.156 / 50$ & 2 & 0.585 & 3.246613 \\
\hline 16 & $2.738 / 135$ & $0.91 / 45$ & $0.01826 / 0.3$ & $0.216 / 139060$ & $0.156 / 50$ & 6 & 1.609 & 4.86992 \\
\hline 17 & $2.738 / 135$ & $0.91 / 45$ & $0.01826 / 0.3$ & $0.186 / 119746$ & $0.156 / 50$ & 5 & 1.399 & 4.638019 \\
\hline 18 & $2.738 / 135$ & $0.91 / 45$ & $0.01826 / 0.3$ & $0.17 / 109316$ & $0.156 / 50$ & 3 & 1.156 & 3.895936 \\
\hline 19 & $2.738 / 135$ & $0.91 / 45$ & $0.01826 / 0.3$ & $0.147 / 11619$ & $0.156 / 50$ & 3 & 0.91 & 3.895936 \\
\hline 20 & $2.738 / 135$ & $0.91 / 45$ & $0.01826 / 0.3$ & $0.123 / 79315$ & $0.156 / 50$ & 2 & 0.631 & 3.246613 \\
\hline 21 & $2.738 / 135$ & $0.91 / 45$ & $0.01826 / 0.3$ & $0.216 / 139060$ & $0.195 / 40$ & 6 & 1.93 & 4.32881 \\
\hline
\end{tabular}


Generation of Heat Transfer Coefficient Data in Regenerator for Stirling Cycle Refrigeration System

\begin{tabular}{l|l|l|l|l|l|l|l|c|c|}
\hline 22 & $2.738 / 135$ & $0.91 / 45$ & $0.01826 / 0.3$ & $0.216 / 139060$ & $0.173 / 45$ & 6 & 1.787 & 4.86992 \\
\hline 23 & $2.738 / 135$ & $0.91 / 45$ & $0.01826 / 0.3$ & $0.216 / 139060$ & $0.156 / 50$ & 6 & 1.6091 & 4.86992 \\
\hline 24 & $2.738 / 135$ & $0.91 / 45$ & $0.01826 / 0.3$ & $0.216 / 139060$ & $0.142 / 55$ & 6 & 1.462 & 4.86992 \\
\hline 25 & $2.738 / 135$ & $0.91 / 45$ & $0.01826 / 0.3$ & $0.216 / 139060$ & $0.13 / 60$ & 6 & 1.379 & 4.86992 \\
\hline
\end{tabular}

Effect of Variation of $\boldsymbol{\pi}$ terms On Experimental Based Heat Transfer Coefficient.
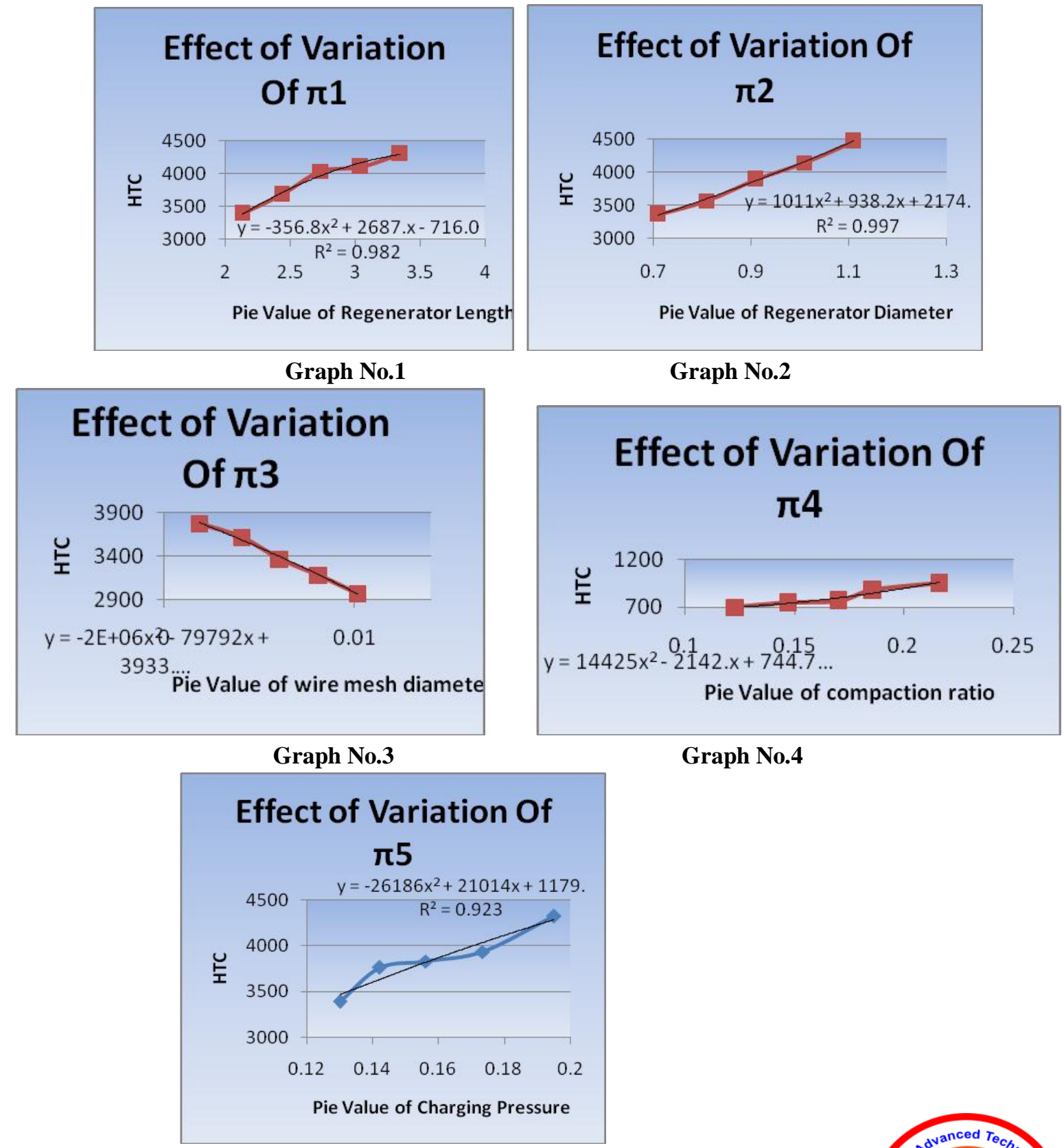

\section{Graph No.4}

Graph No.5 
b) FORMULATION OF POLYNOMIAL MODEL FOR

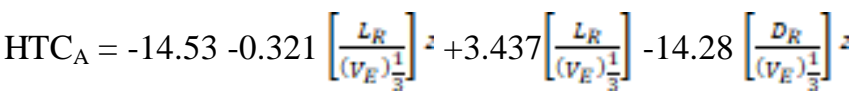

$$
\begin{aligned}
& \left.+30.85\left[\frac{D_{R}}{\left(V_{R}\right) \frac{1}{G}}\right]-16986\left[\frac{D_{M}}{\left(V_{R}\right) \frac{1}{2}}\right]^{2}+10.95\left[\frac{D_{M}}{\left(V_{E}\right)}\right] \frac{1}{2}\right]-36.24\left[\frac{V_{W}}{V_{R}}\right]^{2}
\end{aligned}
$$

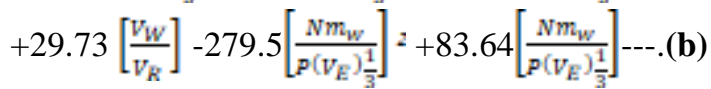

$-14.53-0.321 \pi_{1}^{2}+3.437 \pi_{1}-14.28 \pi_{2}^{2}+30.85 \pi_{2}-16986 \pi_{a}^{2}+10.9$

$5 \pi_{3}-36.24 \pi_{4}^{2}+29.73 \pi_{4}-279.5 \pi_{5}^{2}+83.64 \pi_{5} \quad---(a)$

Effect of Variation of $\boldsymbol{\pi}$ terms On Actual Based Heat Transfer Coefficient.

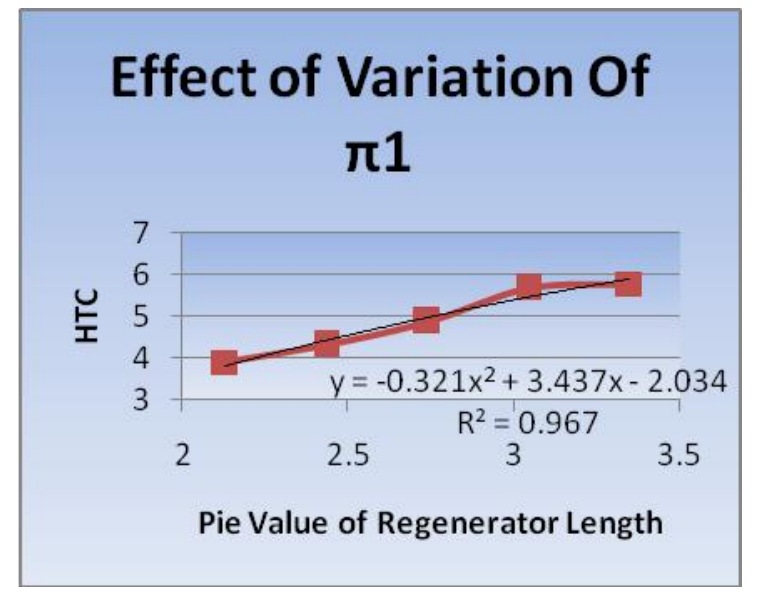

Graph No.6
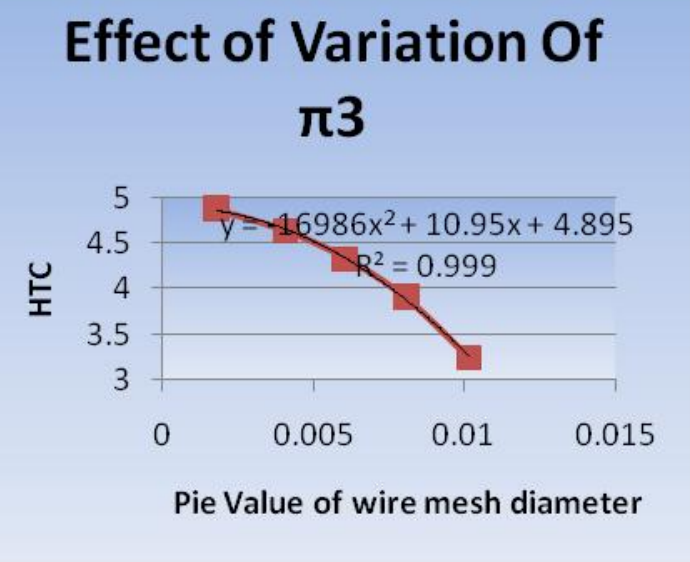

Graph No.8

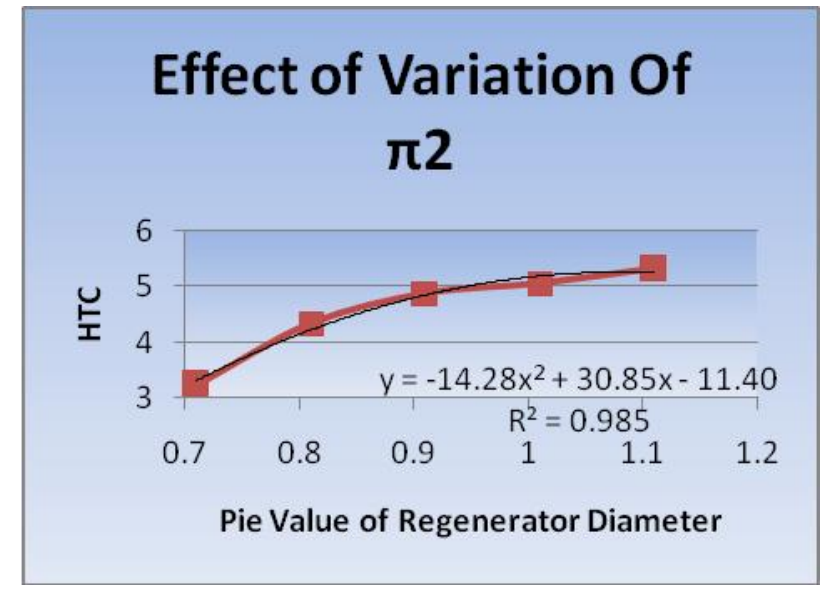

Graph No.7

\section{Effect of Variation Of $\pi 4$}

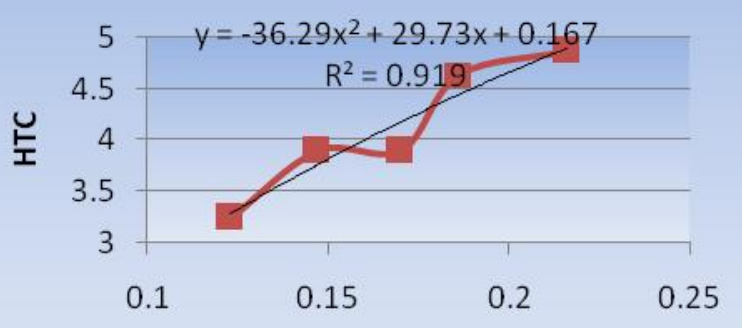

Pie Value of compaction ratio

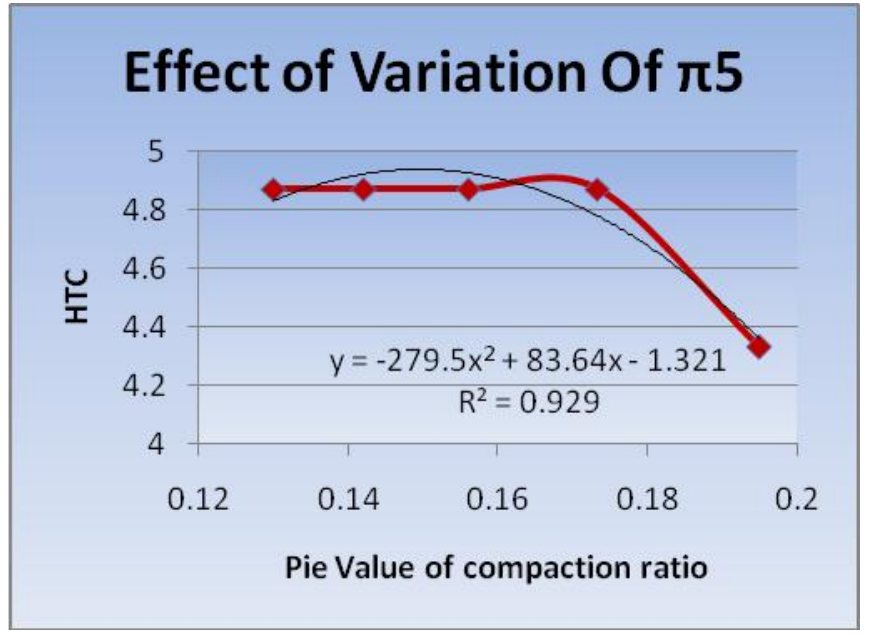

Graph No.9

Graph No.10 


\section{ABBREVIATIONS USED IN ABOVE TABLE AND CALCULATIONS ARE}

LR Length of Regenerator

DR Diameter of

Regenerator

DM Diameter of Mesh Wire

wire

VR Volume of Regenerator

VE Expansion Space Volume

$\mathrm{N}$ Speed of the machine

VM Volume of Mesh

P Charging Pressure

WPower consumed

Temperature

T4 Expansion Space Temperature COPT

Theoretical Coefficient of Performance

COPA Actual Coefficient of Performance

HTCT

Theoretical Heat Transfer Coefficient

HTCA Actual Heat Transfer Coefficient mw Mass

flow rate of water

HTOA Overall Actual heat transfer coefficient HTOT

Overall Temperature based heat transfer coefficient

$$
\begin{array}{rlrl}
\pi 1 & =\mathrm{LR} / \mathrm{VE}^{1 / 3} & \pi 2 & =\mathrm{DR} / \mathrm{VE}^{1 / 3} \\
\pi 3 & =\mathrm{DM} / \mathrm{VE}^{1 / 3} & \pi 4=\mathrm{VM} / \mathrm{VR}
\end{array}
$$$$
\pi 5=\mathrm{Nmw} / \mathrm{PVE}^{1 / 3}
$$

COPTE $=$ experimental temperature based Coefficient of

Performance

COPA $=$ theoretical temperature based Coefficient of

Performance

\section{CONCLUSIONS}

1) It is observed that evaluated theoretical coefficient of performance by assuming the heat transfer in regenerator takes place by convection and experimentally COP are different and varying by large range i.e 40 to $70 \%$.

2) To improve the performance of model, Heat transfer coefficient must be modified by to account for heat transfer in a regenerator by all three modes i.e conduction, convection $\&$ radiation.

3) It is established that as the length of the regenerator goes on increasing the actual Heat Transfer Coefficient and temperature based Heat Transfer coefficient both are increased. The actual heat transfer coefficient is maximum when regenerator length $=5.353 \times \mathrm{V}_{\mathrm{E}}{ }^{1 / 3}$.

4) It is established that as the compaction ratio (i.e. volume of wire mesh in regenerator / regenerator volume) heat transfer coefficient of performance both are increased. The actual coefficient of performance is maximum when compaction ratio (i.e. volume of wire mesh in regenerator / regenerator volume) is 0.410 .

\section{REFERENCES}

1. G Thombare, D.G, S.K. Verma" Technological development in the Stirling cycle engines. Renewable and sustainable Energy Reviews”, 12, 2008, pp. $1-38$.

2. G. Walker "Stirling-cycle machines" Book, Oxford, Clarendon Press, 1973 ix, pp156

3. Nitesh B Umale,DhananjayThombre "Theoretical Analysis of Effect of Regenerator Geometry and Material on Stirling Engine Performance", conference paper, june 2015 pp 38-43

4. M. Tarawneh , F. Al-Ghathian "Numerical Simulation and Performance Evaluation of Stirling Engine Cycle" Jordan Journal of Mechanical and Industrial Engineering Volume 4, Number 5, November 2010 ISSN 1995-6665 pp $615-628$

5. S.K.Choudhary et.al "Design and performanceof an Eco-Friendly Refrigeratio System working on stirlingcycle'International Conference at deptt of MechEngg.on Energy and Environment,Faculty of Engg\&Technology,JamiaMillia Islamic New Delhi 23-24 JAN 2004 pp 496-500.

6. S.K.Choudhary “ Experimental Optimization of some sub systems of stirling cycle refrigeration with Non CFC Gases" Thesis, April 2010 